\title{
La géographie juridique. Recherche et limite d'une définition
}

The "legal geography". Research and limited definition

Jean-François Boudet

\section{(2) OpenEdition}

Journals

Édition électronique

URL : http://journals.openedition.org/developpementdurable/10744

DOI : 10.4000/developpementdurable.10744

ISSN : 1772-9971

Éditeur

Association DD\&T

Référence électronique

Jean-François Boudet, « La géographie juridique. Recherche et limite d'une définition », Développement durable et territoires [En ligne], Vol.6, n¹ | Mars 2015, mis en ligne le 31 mars 2015, consulté le 01 mai 2019. URL : http://journals.openedition.org/developpementdurable/10744 ; DOI : 10.4000/ developpementdurable.10744

Ce document a été généré automatiquement le 1 mai 2019.

Développement Durable et Territoires est mis à disposition selon les termes de la licence Creative Commons Attribution - Pas d'Utilisation Commerciale 4.0 International. 


\title{
La géographie juridique. Recherche et limite d'une définition
}

\author{
The "legal geography". Research and limited definition
}

Jean-François Boudet

1 Nous voici aujourd'hui bien désorientés, hésitant entre la proximité familiale et amicale qui rassure et la globalité qui effraie, entre la chaleur et la fureur universelle (Guillebaud, 2003). Comme si les espaces physiques et les paysages imaginaires, globalisés eux aussi, broyés par la vitesse ou dissous derrière les écrans, ne s'emboîtaient plus dans nos têtes. Comme si, à ne plus mesurer le proche et le lointain, nous dodelinions, interdits.

2 Quartiers sans âme, communes avec ou sans communautés, régions bousculant les nations, nord-sud sans est-ouest, Europe ravaudée et sans outre-mer, tiers-monde englouti, identités en errances, réfugiés climatiques, pôles en liquéfaction, continents sous le choc de la mondialisation et de la finance ou du tout numérique, tout s'est bien éparpillé et désaccouplé (Dupuy et Lavigne, 2009) : la géographie se dérègle autant que son histoire, le pouvoir "déterritorialise " pour justifier son impuissance à gouverner (Martens, 2009, p.9). Nous sommes rentrés dans l'ère de la vitesse et des grandes migrations. À cet égard, le resserrement de la planète oblige à penser en termes mondiaux et met en cause le concept de souveraineté étatique. L'ordre souverain est dépassé dans l'espace et dans le temps, il doit s'adapter à des logiques qui s'entendent désormais dans des espaces de concurrence ou d'extranéité (Cenk Keskin, 2010).

Entre ce qui doit être (dans le domaine normatif) et ce qui est (dans le domaine de l'espace), tout est alors affaire de nuances qui repoussent, qui rassemblent, qui unissent ou qui intensifient les liens et les dialogues. Ces deux systèmes de pensées que sont les sciences juridiques et géographiques contribuent en ce sens "au développement d'une interface géo-légale, lieu de production d'un socle théorique commun aux deux disciplines " à travers cette notion de géographie juridique (Forest, 2009, p. 23 ; Cavaillé, 2009, p. 45 ; Perret, 2009, p. 261). Pourtant, le dialogue entre géographes et juristes peut conduire à parler de "géographies du droit» plutôt que de "géographie du droit» tant les approches peuvent être soit critique ou inclusive, soit positiviste ou spéciale. À cet égard, 
les développements du courant anglo-saxon de la "legal geography" conduisaient à plutôt développer une approche pluridisciplinaire qu'interdisciplinaire, se limitant le plus souvent à quelques citations de lois ou quelques descriptions géographiques du territoire. Les études critiques outre Atlantique adoptées dans les années 1980 et 1990 ont pu montrer que la géographie juridique ne se distingue pas de l'étude générale du droit par ses aspects méthodologiques mais plutôt par sa perspective spatiale dans l'étude des concepts et de la formulation des problématiques (Forest, 2009,p. 23).

C'est pourquoi cette interdisciplinarité admet au préalable des postulats qui, de facto, limitent la définition de la géographique juridique. Ne conviendrait-il pas dans ces conditions de favoriser l'étude du quatuor " société, droit, nature et espace "? Dans cette optique, il s'agirait alors de s'intéresser à la matérialité du droit à travers l'inscription de celui-ci dans et à l'espace géographique (Forest, 2009,p. 24). Cette approche ambitieuse et empirique dépasserait la simple étude de la géographie juridique pour s'amplifier au travers d'une génétique juridique. Cette orientation permettrait surtout de proposer une théorie de la géographie juridique à travers l'économie politique.

\section{De quelques postulats pour essayer de définir la géographie juridique}

\subsection{Le constitutionalisme et la démocratie cosmopolitique aux fondements de la géographie juridique}

5 Le premier postulat s'inscrit dans le concept même de "constitutionnalisme " ou de « démocratie cosmopolitique ». Il conduirait à croire que les conflits ne pourraient être résolus au niveau mondial que par le biais de dispositifs juridiques et légitimes (Archibugi, 2009). Pourtant, le lien unissant les individus membres d'une communauté est essentiellement d'ordre affectif alors que celui qui unit les individus au sein des sociétés se situe amplement au niveau des intérêts. Les notions de "communauté " et de "société " peuvent toutefois se chevaucher: une communauté n'est pas toujours une communauté pure, il y a toujours des intérêts qui s'y mêlent. À l'inverse, une société n'est jamais une société pure, il y a toujours des liens affectifs qui se créent quand une société se développe. Bien que la notion de frontière n'ait plus le même sens qu'auparavant, les relations internationales ne peuvent alors être que transnationales (De CoudhenoveKalergi, 1988). Les notions de "communauté internationale " (Kant, 1784), de «village mondial» (Mc. Luhan, 1967) ou de "économies monde» (Braudel, 1979 et 1985) sont totalement fausses.

6 On crée d'ailleurs des conférences "géographiques", "financières " ou "environnementales», déférant aux désirs des puissants, réunissant les principaux explorateurs d'un continent afin de coordonner les moyens et les activités mondiales. On s'impose, on apporte des moyens de communications, on prospecte, on étudie et on recueille : anthropologie, ethnologie, cartographie, géologie, biologie... On contracte, on institutionnalise des rapports entre puissants, on se partage les terres entre puissants. Le tout se justifiait hier par une pensée universaliste missionnaire (Chakrabarty, 2010). Le droit d'ingérence humanitaire est souvent invoqué depuis la chute du mur de Berlin pour justifier désormais des interventions militaires occidentales. Voilà qui rappelle le vieux discours colonial sur la «mission civilisatrice ». Il devint rapidement clair pour les États 
dominateurs que l'occupation des territoires devait être également plus systématique et plus rationnelle : on distinguait alors les colonies d'exploitation de celles de peuplement. Chacun y avait sa cause, chacun défendait son «pré carré » (Fiero-Domenech, 1986). L'objet juridique de cette organisation n'était que normatif, c'est-à-dire qu'il ne s'appliquait qu'aux seules institutions ou aux autres modes de gouvernement dans un milieu relativement homogène créant à terme une nouvelle fracture territoriale. Cette occupation tend, en quelque sorte, à identifier le meilleur des mondes ou la meilleure des espèces de domination politique (Saint Augustin, Platon, Aristote ou plus tard l'historien arabe Ibn Khaldun ou Montesquieu).

\subsection{La politique et la géopolitique aux fondements de la géographie juridique}

7 Cette recherche originale d'« espaces communs » sous-tend également la conviction selon laquelle la vérité ne peut apparaître que par la multiplication des points de vue complémentaires sur un même objet d'étude : un droit lié au politique et au géopolitique, un droit lié à l'histoire, un droit typiquement territorialisé, un droit cadré par les frontières et conditionné par la volonté propre des États. La formule romaine "Ubi societas, ibi jus » est à cet égard restée célèbre: elle suggère qu'une société qui a suffisamment de consistance, quelle que soit sa taille, se donne un droit. Droit étatique et système juridique ne sont pourtant pas identiques puisque plusieurs systèmes de droit peuvent coexister dans un État et un système de droit peut s'appliquer dans différents États (Troper, 1986, p. 28). Les systèmes juridiques, quel que soit le niveau de pluralisme territorial qu'ils consentent, ne supportent cette différenciation à base territoriale que dans certaines limites dérivant de principes qui fondent leur unité : droits fondamentaux, libre-circulation, appartenance à un peuple unique, principes économiques... Les territoires ont dès lors une fonction indispensable qui est celle d'être des «vecteurs de différenciation du droit » (Auby, 2006, p. 17) et non du maintien d'égalités territoriales (variable de genre dans la création ou la destruction d'emploi, découplage de la progression de l'emploi public et de la croissance démographique, poids des pensions de retraite et du choix de résidences des retraités, etc.).

Cette recherche, empruntée à la méthode scientifique de la géographie générale, se décompose en une collecte, un classement, une sélection de données juridiques puis par la mise en place d'une cartographie de ces données, cartes qui pourront être par la suite interprétées et discutées. Est d'ailleurs mis en place en France un Institut géographique national (IGN), établissement public administratif créé par un décret du 27 juin 1940, dont la mission est d'assurer la production, l'entretien et la diffusion de l'information géographique de référence en France. Cette matière ainsi développée se définirait par le terme peu courant de géographie juridique: certains systèmes de droit ne sont pas seulement importants en eux-mêmes pour des raisons historiques, politiques, économiques, sociales, techniques, mais le sont également parce qu'ils aident à comprendre - et ils sont nombreux - qui les ont transposés ou imités dans une certaine mesure. À cet égard, on distingue traditionnellement cinq « grands » modèles (sans que la doctrine comparatiste en soit d'accord sur le nombre) :

- le système de droit romano-germanique ;

- le système de "common law";

- le système mixte ; 
- le système de droit coutumier ;

- le système de droits musulmans.

9 Cette diversité de modèles est la traduction d'une divergence dans la vision du droit dans la société. Essayer de saisir les raisons de cette diffusion, c'est répertorier, classer les pays et en établir un planisphère mondial. Entre génotype et phénotype (Sacco, 1991), entre circulation de "grands systèmes de droit » et celle de "familles de droits » (David et Jauffret-Spinosi, 2002), la géographie juridique serait plus précisément «la science de l'organisation juridique de l'espace terrestre par l'homme » (Parra, 2007, p. 27). Ce choix de chacun est toujours discutable, sans doute arbitraire et réducteur, car toute classification porte en germe les défauts de ses qualités (Husa, 2004, p. 11 et Wald, 2005, p.187). Certains préféreront alors raisonner en fonction de "cultures juridiques » (Cappeler et Kitamura, 1998), d'«aires culturelles» (Thiebault, 2006, p. 237) ou de «traditions juridiques» (Gleen, 2010).

\subsection{La collaboration et la comparaison aux fondements de la géographie juridique}

10 Ces démarches méthodologiques - éclairantes soient-elles - ne sont pas non plus en ellesmêmes satisfaisantes car elles accentuent un domaine (sociologie, économie, droit, culture...) que la géographie juridique réfute en tant que comparaison plus synthétique des systèmes de droit. En effet, comme le soulignait David, la géographie juridique ouvre à la collaboration scientifique qui s'efforce à " redécouvrir [dans la recherche des effets et des causes] l'universalité de la pensée humaine et la synthèse qui constitue toute cuvre de civilisation » (David, 1966, p. 1738). Ce n'est donc qu'à travers les influences réciproques de la géographie et du droit qu'on pourra discuter de "géographie juridique». Cette question a été peu étudiée. Les juristes se contentent en général de citer un passage de l' Esprit des Lois pour simplement régler les rapports sociaux et délaisser l'étude des rapports entre géographie et droit (Montesquieu, 1860).

- Livre quatorzième : Des lois dans le rapport qu'ils ont avec la nature du climat ;

- Livre quinzième : Comment les lois de l'esclavage civil ont du rapport avec la nature ;

- Livre seizième: Comment les lois de l'esclavage domestique ont du rapport avec la nature du climat ;

- Livre dix-septième : Comment les lois de la servitude politique ont du rapport avec la nature du climat ;

- Livre dix-huitième : Des lois dans le rapport qu'elles ont avec la nature du terrain.

11 S'inscrivant dans la thèse de Montesquieu, le projet scientifique de la géographie juridique peut alors paraître très modeste puisque c'est admettre que le climat et le terrain créent de grandes différences dans le " caractère de l'esprit ", les "passions du cœur » et les « lois » (c'est-à-dire les coutumes, les mœurs, les lois proprement dites). En conséquence de quoi, le climat et le terrain doivent s'accorder à ces différences pour permettre aux décideurs publics (agents publics et personnels politiques) de développer des politiques foncières (donc un droit foncier), des politiques urbanistiques (donc un droit de l'urbanisme) et, pourquoi pas, une politique d'aménagement du territoire (à travers un droit de l'aménagement du territoire). La science de la "géographie juridique " s'attache ainsi à rechercher les causes d'ordre géographique agissant sur l'homme et façonnant la société et, inversement, à convenir des règles juridiques influencées par la géographie. Cette méthode de travail doit cependant être globalement 
combattue parce qu'elle relève d'un certain déterminisme physique et l'énumération serait pur exercice de rhétorique. Elle ne doit être qu'un outil d'arbitrage juridique de remèdes aux blessures territoriales (Gottmann, 1952, p. 29). Reste que la géographie moderne embrasse la mondialisation comme sphère d'un monde globalisé, et cherche à comprendre notre planète et toutes ses complexités humaines et naturelles du passé, du présent et de l'avenir. En tant que "pont entre les sciences humaines et physiques », la géographie moderne a sans conteste une influence égale sur le droit. Elle tend en effet à mettre en lumière les régularités et les ressemblances entre les espaces pour énoncer des lois générales explicatives (par des hypothèses et par des échelles) (Debie, 1995 ; Charvet et Sivignon, 2002). Le droit ne peut pas être indifférent à cette évolution. Certains soutiennent à cet égard la pertinence de la notion traditionnelle de « familles de droits " pour actualiser les classifications de la géographie juridique (Legeais, 2008, p. 92).

Première conclusion : cette pédagogie trouve rapidement sa limite car la mondialisation a également des effets sur le droit. En effet, l'internationalisation des échanges commerciaux accomplie sur plusieurs décennies puis la transnationalité des investissements directs effectuée au cours des années 1970 ont laissé transparaître une globalisation des économies dans les années 1980. Cette dernière signifie la mise en réseau (positive) des circuits de production et de communication (et donc du droit) et la montée (réactive) des intégrismes identitaires.

13 Complexes, hasardeuses, les classifications juridiques en groupes et sous-groupes perdent alors rapidement leur légitimité pour mettre davantage en avant les «réseaux» et solidarité mécanique. Mieux vaudrait alors anticiper ces échelles pour considérer le dépassement - ambitieux - de la notion de géographie juridique par celle de génétique juridique (Legeais, 2002, p. 87).

\section{De la génétique juridique pour dépasser le cadre limité de la géographie juridique}

\subsection{La génétique juridique, vecteur de modélisation}

14 La sociologie empirique du droit considère la compréhension des faits normatifs par l'observation de la société internationale (Saidon, 1993). En ce sens, le mode de gouvernement adopté par les colonisateurs a évidemment influencé le devenir des excolonies (Cogneau, 2007). Cependant, plusieurs des principaux États montrés en exemple pour leur vitalité démocratique - Afrique du Sud, Botswana, Namibie, Zambie, Ghana appartiennent à l'aire anglophone, tandis que les pays francophones stagnent ou régressent à l'image de la Côte d'Ivoire, du Mali ou de la Centrafrique.

Le point de départ n'est pas le même : le colonisateur français a plus souvent envahi des régions islamisées et peu peuplées, le britannique s'est fréquemment attribué des lieux plus propices à la colonisation de peuplement. Le type de gouvernement a ensuite divergé : l'indirect rule" britannique s'est en effet appuyé sur les élites locales par opposition au jacobinisme français formant ses fonctionnaires dans un monde commun. Tandis que la France privilégiait l'école laïque et en langue française, la Grande-Bretagne subventionnait enfin l'enseignement confessionnel pratiqué par les missionnaires, plus souvent en langue vernaculaire. À l'indépendance, ces deux modèles avaient forgé des élites différentes (fonctionnaires en Afrique francophone et professions libérales en 
Afrique anglophone). Cette divergence se traduisait dans le modèle politique : la France a mené une décolonisation en trompe-l'œil à travers des présidents vassaux qui a assuré une stabilisation politique et une certaine prospérité économique jusqu'à récemment. Dans le même temps, les pays anglophones vivaient douloureusement leur «crise d'adolescence » qui débouche aujourd'hui sur une plus grande autonomie. Ce sont ces filiations et modèles primaires qu'il convient de qualifier de " génétique juridique ».

\subsection{La génétique juridique, vecteur de standardisation}

Cette notion fait alors davantage percevoir la géographie juridique limitée à une simple stratégie de cartographie des espaces juridiques et de clonage des gênes antérieurs à l'identification de leurs " produits ». Le caractère artificiel de l'ordonnancement juridique ne constituerait pas un obstacle insurmontable à la naissance d'une identité : on pourra alors prétendre à enseigner des sous-thématiques de la géographie juridique comme par exemple la "géographie administrative" (parmi d'autres: Dupont-Ferrier, 1930; Bertrand, 1974 ; Gyselen, 1989 ; Ould el Hacen, 1989), la " géographie constitutionnelle » (parmi d'autres: Dorion, 1980, p.69; Ferreira da Cunha, 2009, p. 31 et 2010; Sy Mouhamadou, 2007, p. 453) ou le «droit géographique » (parmi d'autres : Bodichon, 1866, p. 163 ; Kalampalikis, 2007, p. 190 ; Chauveau, Jul-Larsen, Chaboud, 2000, p. 137 ; Espinas, 1931, p. 394 ; Schroter, 2007, p.663). Il ne s'agit cependant pas d'une discipline scientifique particulière mais de rapports filiaux entre les mots hégémonie et normes ou entre ceux de standards juridiques et normes. Dans le premier cas, le mot hégémonie justifie les postulats de la génétique juridique puisqu'il prend le sens de domination d'un État par sa puissance politique, militaire, économique et culturelle sur d'autres États. Cette hégémonie n'est cependant pas obligatoirement absolue et peut correspondre à des situations où le pouvoir est distribué de manière inégale entre les différents pays. On pourrait donc tout aussi bien employer selon les époques et les territoires, les termes de "prééminence », de "suprématie ", d'« empire» ou d'« impérialisme ", lesquels conviennent uniformément pour évoquer la notion de puissance et de son exercice. La question de l'exportation d'un système juridique sur un autre devient alors légitime mais perd rapidement de sa pertinence parce qu'elle doit prendre en considération des données extra-juridiques et le système ainsi importé doit vivre en dehors de son modèle.

On pourra encore développer aujourd'hui cette notion a-juridique de " gouvernance » qui trouve à se multiplier à outrance dans le vocabulaire des administrations (Dubus, Helle et Masson-Vincent, 2010). Elle était déjà passivement introduite à travers le phénomène américain de standardisation ("standardization" en américain) et la notion de standard juridique. Associé aux règles et normes en matière commerciale et financière dans la théorie anglo-saxonne, l'approche outre Atlantique consiste à définir dans un premier temps des communautés d'intérêts, à en déduire les standards dans un second temps et à définir dans un troisième temps les services adéquats. Ce décalage est encore perceptible dans le partage du pouvoir politique : la conception française enseigne que ceux qui sont démographiquement majoritaires doivent gouverner par opposition à la conception anglo-saxonne du « one man, one vote » qui s'impose sur le continent de l'Afrique de l'Est à l'Afrique du Sud. Cette structuration permet alors d'acquérir une certaine domination par la connaissance scientifique et consiste aussi à évacuer toute grille de lecture politique d'événements nationaux ou géopolitiques en Afrique (Nouschi, 2007, not. 315 et 468). L'approche européenne est différente et plus récente. Il s'agit tout d'abord de 
différencier standards et normes, la première notion n'étant pas nécessairement une norme mais pouvant le devenir. Les européens sont par ailleurs demandeurs de rationalisation, notamment en matière économique mais considèrent dans le même temps le procédé de standardisation comme une entrave à la liberté du commerce et comme une atteinte à l'individualisme. Bref, les balises des standards sont diffuses, la technique étant fondée sur la normalité et les critères restant subjectifs. Elle reste donc indéterminée (Bernard, 2010).

\subsection{La génétique juridique, vecteur de standardisation}

Cet artifice dépasse simplement le droit et la géographie car cette construction scientifique de la géographie juridique n'a été en elle-même fondatrice d'identités. À cet égard, si les identités régionales françaises ont été reconnues dès la loi du 2 mars 1982 (art. L. 4221-1 CGCT), elles n'ont pas spontanément généré un sentiment d'appartenance chez leurs habitants (à l'exception notable des régions historiques de la Corse, de la Bretagne ou de l'Alsace). L'explication réside dans le seul fait qu'à leur origine, les collectivités régionales françaises n'ont pas été créées dans une perspective historique et affective mais dans un but économique et utilitariste. De même, si Musulmans, Chrétiens et Juifs peuvent très facilement se retrouver dans la religion du Livre, il en est différemment avec la doctrine philosophique et religieuse du chinois Confucius ou avec la religion dualiste fondée par Zarathoustra et professée de nos jours par les Parsis (Raffestin, 1985, p. 101 et Pomeranz, 2010). De même, le véhicule de la langue ne permet plus véritablement d'exprimer une filiation entre espaces territorialisés et juridiques comme pouvait l'être en son temps la langue d'Oc et la langue d'Oïl. Le « citoyen monde » parle volontiers le «globish» pour communiquer facilement dans le monde entier. Son vocabulaire de 1500 mots bien choisis et sa syntaxe rudimentaire permettent de discourir aisément avec tout interlocuteur exotique (Nerrière, 2004). S'il reste le meilleur moyen de faciliter la communication les échanges entre non-anglophones, il appauvrit surtout les langues dans leur sens littéraire et peut être source de nombreuses violences.

19 Appartenant au vocabulaire du «choc des civilisations » et de la "guerre froide " (à travers la théorie des «blocs de l'Est et de l'Ouest »), la géographie juridique n'entend pas rechercher l'interface entre ordres juridiques parce que l'objet politique déforme ou transforme tout simplement l'espace social et juridique (Wijffels, 2008, p. 228). Le passé était marqué par l'européocentrisme qui conçoit la société internationale comme un objet immobile que l'Europe avait pour mission d'intégrer à l'histoire (du Progrès) (Bridel, 1908). Le XXI ${ }^{e}$ siècle introduit les reculs de l'approche comparative eurocentriste, de l'hégémonie privatiste et du positivisme législatif et montre les difficultés à envisager une discipline comparatiste susceptible de transcender les prémisses apparemment inconciliables d'ordres juridiques différents (Wijfells, 2008, p. 230). La génétique juridique pourrait alors dans ce cadre être fort utile pour essayer de définir une forme de géographie juridique au XXIe siècle.

Deuxième conclusion : à l'inverse de la géographie juridique, le projet ambitieux de la génétique juridique parait propice à comprendre les liens contemporains entre ce qui doit être (dans le domaine normatif) et ce qui est (dans le domaine de l'espace). L'ensemble de ces éléments permet de penser que l'économie politique, entendue comme l'étude des besoins, de l'organisation de la production, de la circulation des richesses et de 
leur répartition, soit le seul schéma d'interprétation de la réalité concrète de la géographie juridique au XXI ${ }^{\mathrm{e}}$ siècle.

\section{L'économie politique, schéma unique d'interprétation de la réalité concrète de la géographie juridique}

\subsection{La mise en place d'imitations et d'espaces harmonisés}

21 La transformation économique majeure depuis les années 1980 est la mondialisation de l'économie. Ce vocable sert à désigner le processus d'interdépendance de plus en plus prononcée des économies nationales découlant de la création d'un marché planétaire pour les marchandises, les services, les capitaux, mais aussi l'information, les idées, les produits culturels et médiatiques. Dans ce nouvel environnement, penser en termes d'entreprises, de technologies ou d'industries, voire d'économie nationales est anachronique. L'économie politique est alors un vecteur favorable à une définition moderne de la géographie juridique. Des économistes ont rapidement montré leur intérêt par certains aspects de la localisation et des avantages comparatifs régionaux. Il y a également eu une forme de mimétisme des données économiques et une forme d'échanges de procédés juridiques et financiers, telle la taxe sur la valeur ajoutée (TVA) qui s'est progressivement imposée dans de nombreux pays comme le mécanisme de taxation préféré pour les biens et les services (Mehl et Beltrame, 1984, p. 635). G. Tarde, juriste et sociologue français de la seconde moitié du XIX ${ }^{\mathrm{e}}$ siècle, a pu ainsi développer ces "lois de l'imitation », théorie selon laquelle les innovations ou les découvertes couvrant tous les aspects de la vie sociale (religieux, politique, juridique, scientifique, économique, linguistique et culturel) peuvent n'être qu'un perfectionnement, si faible soit-il, d'innovations réalisées auparavant (Tarde, 1895). Ces «initiatives rénovatrices » se propagent ensuite par imitation et répétition, s'étendent d'un milieu social vers un autre, d'un village à un autre, d'un pays à un autre. Les civilisations conquérantes imitent ainsi les civilisations conquises et vice-versa. Il y a seulement échanges de décisions, de dispositions, de mesures, la matière juridique ne faisant pas exception.

Cela peut laisser supposer que les frontières politiques des États s'affaiblissent dans les faits au profit d'un monde sans frontières, d'une intensité des circulations des flux, de l'idéologie du marché sans barrières et du "market access" et de l'uniformisation de la norme (Wallon-Leducq et al., 1988). Il ne faut cependant pas se tromper de débats car la production de frontières politiques s'est en réalité accélérée depuis deux décennies, notamment avec l'accession à l'indépendance de territoires sans compter les pratiques de durcissement des frontières (dites "fencing") depuis septembre 2001 (Foucher, 2007 et Thual, 2009, p. 46). Ces frontières politiques doivent être comprises ici comme des institutions créant des effets symboliques de représentations et comme supports de cartes mentales répondant à un besoin d'identité séparant le dedans du dehors. Il n'y a donc pas de contradiction entre cette production et la mise en place d'imitations ou d'espaces harmonisés. Ce raisonnement confirme toute réflexion sur les frontières politiques en termes de géographie juridique. Elle le dépasse. À cet égard, la mondialisation s'esquisse bien avant l'époque contemporaine (Chanda, 2010). Elle s'amorce avec les Grandes Découvertes de la fin du XVe siècle qui permettent l'essor du commerce transatlantique entre l'Ancien et le Nouveau Monde et entraînent la naissance 
du grand capitalisme marchand et de l'économie politique, en même temps que le développement des États-Nations.

\subsection{La mise en place d'une « économie-monde »}

Cette orientation scientifique relève autant des grands penseurs économiques (notamment autour d'A. De Montchrestien, d'A. Smith, de P. Samuelson ou de R. Coase) que philosophiques (notamment autour de J. Harsanyi, de J. Rawls, d'A. Downs, de K. Aarrow, d'A. Sen ou de M. Olson). L'économie politique peut décrire et analyser l'activité économique par rapport aux données politiques, en essayant d'expliquer le fonctionnement et de trouver les lois qui régissent l'activité économique par rapport à l'action des pouvoirs publics (en anglais "political economy"). Elle peut également appliquer les outils de l'analyse économique à un système politique donné dans le but d'expliquer le choix des politiques publiques en fonction des préférences des agents et des objectifs propres des dirigeants (en anglais, "public choice"). Cet emprunt des sciences économiques peut aider à définir le concept de "géographie juridique». En effet, c'est bien le développement de l'« économie monde » à travers la révolution des transports terrestres, maritimes et aujourd'hui technologiques qui a permis les plus grandes migrations, la configuration de géographies des techniques, d'économies, des langues, des religions et des valeurs et la mise en place d'espaces harmonisés. La géographie juridique serait alors la science et l'étude de cet agencement d'espaces autour de principes et de règles communs, l'un pouvant être imbriqué dans un autre. Le droit anime cette analyse structurelle et fonctionnaliste en tant qu'ensemble des règles qui régissent les rapports en société, c'est-à-dire en considérant un «ensemble des modèles d'organisation et d'interrelation, des individus et des groupes, des associations, des organisations et des institutions qui concourent à la satisfaction concertée des besoins de la collectivité»(Fichter, 1965, p. 94).

Cette construction scientifique prend alors en considération dans un premier temps la totalité sociale comme idéologiquement intelligible, puis dans une seconde éventualité le besoin en termes de valeurs économiques. Ce phénomène de mondialisation bouleverse les sociétés industrielles, modifie la nature des emplois et aggrave les inégalités, car qui dit sociétés ouvertes, dit sociétés dépendantes de l'exercice qu'une myriade d'individus font sur cette liberté. Il corrige intrinsèquement les principes et les postulats précités de la géographie juridique : l'économie politique domine et explique la construction de ces espaces harmonisés de droits. La science de la géographie juridique est renouvelée par la mise en place d'espaces harmonisés et d'institutions se retrouvant autour d'un principe, d'une méthode, d'un outil juridique, d'une politique, d'une croyance, d'une langue ou d'une industrie dominants parmi d'autres. Le "commerce équitable» entre également dans cette logique puisqu'il entend développer des relations privilégiées et équilibrées avec des groupes de petits producteurs défavorisés dans les pays du Sud et promouvoir leur développement durable grâce à la commercialisation de leurs produits, suivant des conditions commerciales avantageuses sur les marchés développés. Le mouvement anti, puis altermondialiste qui dénonce la globalisation financière et la mondialisation des investissements et des entreprises multinationales fédère différents mouvements nés dans les années 1990 et s'organise aussi dans une charte « des principes du forum social mondial». économique, en prenant "radicalement" au sens strict du terme, c'est-à-dire que sa racine 
est très exactement économique ». On a pu alors militer "Pour une géographie juridique ", cette notion étant définie comme la "science des effets spatiaux comparés de l'utilisation des normes juridiques à support spatial définies, au moins pour partie, au niveau local» (Perret, 1994, p. 520). À la vérité, toute communauté humaine a besoin d'une autorité politique, quelle que soit sa dénomination. Si l'État national ne peut plus être le seul cadre de référence territorial qui permet d'apporter cette réponse politique à la mondialisation des économies, un autre cadre s'impose et peut se manifester dans cette expression de géographie juridique. En effet, l'économie s'est affranchie du jeu de pouvoir territorial dont elle était prisonnière en développant de nouvelles stratégies de pouvoir dans l'espace. Pour ce faire, elle a multiplié les ordres spécialisés pour assembler le territoire, l'autorité et les droits (Sassen, 2009).

\subsection{La mise en place d'un « pluralisme ordonné »}

Cette construction d'ordres spécialisés part de l'hypothèse que la globalisation économique ne pose pas un problème d'échelle juridique mais qu'elle emporte des transformations fondamentales de la nature et de la forme de régulation, des modes d'élaboration et de mise en œuvre des normes. Le Professeur Delmas-Marty soutient en ce sens la thèse du "pluralisme ordonné ", selon laquelle le droit peut réussir à agencer la complexité du monde en mouvement sans pour autant la supprimer mais en la transformant. Cette théorie donnerait une consistance et un support doctrinal à la science de la géographie juridique. Il y a tout d'abord des processus d'interaction qui sont «le reflet des divers pluralismes ». Ces derniers s'opposent à l'extension d'un système à vocation hégémonique. La base normative commune est assurée par la cohérence de règles adaptées à une échelle territoriale par entrecroisements d'autres principes ou valeurs. Les niveaux d'organisation ne peuvent ensuite être que territoriaux et l'organisation juridique se situe pour l'essentiel au niveau des États. Elle peut soit les dépasser, soit les intégrer dans cet espace par convergences ou fragmentations politiques. Le troisième élément réfléchit les vitesses de transformations du droit, ses rythmes et ses dynamiques. Il peut y avoir ici «asynchronie» (« différents espaces, différentes vitesses ») ou "polychromie» (« un espace, plusieurs vitesses»). Le «pluralisme ordonné » met alors en place un droit «à la carte» s'appuyant sur l'articulation des différents pouvoirs politique, juridique et économique. Cette construction reflète totalement la géographie juridique comme lieu, corps et ensemble de rencontres des géographes et des juristes.

Ces figures juridiques renouvelées, voire combinées, d'espaces géographiques semblent compenser les travers de la mondialisation contemporaine et peuvent, à certains égards, expliquer le désordre mondial contemporain. Elles demandent une responsabilité équitable des acteurs juridiques et politiques à une échelle planétaire et impliquent une très forte pondération des rapports de force entre les États et ces espaces. Cela sous-tend également un refus des États de succomber à la tentation hégémonique au bénéfice du pluralisme et une volonté temporelle et spatiale de coopération. Il pourrait être alors reproché à cette théorie du " pluralisme ordonné » de poser les bases d'un idéal opposé à d'autres traditions, ce qui conduirait naturellement à une forme d'hégémonie. La géographie juridique ne refléterait alors que l'étude d'une idéologie orchestrée sur un territoire donné (Brimo, 1986, p. 68). 
isième et dernière conclusion: cette déconstruction de la géographie juridique permettrait surtout de valoriser la jonction des sens juridiques et géographiques et, en conséquence, de bâtir un schéma d'interprétation indiscutable de la matière retrouvée : la géographie juridique se définirait comme l'étude scientifique de l'agencement d'espaces harmonisés par le droit, étant entendu que ces territoires géographiques et juridiques peuvent s'imbriquer sans en être exclusif. Il s'agirait d'étudier plus exactement le « pluralisme ordonné » dans sa vivacité géographique et juridique.

Cela étant, entre le concret et le figuré, il existe un point de rencontre que Bachelard situe dans l'imaginaire car, dit-il, "on ne trouve pas l'espace, il faut toujours le construire" (Bachelard, 1937, p.15). Le concept de géographie juridique est sans cesse dans ce balancement qui laisse sa place lui aussi à un imaginaire juridico-politique ou plus exactement à l'énoncé d'une idéologie. La conclusion de ce travail ne peut aller que dans ce sens.

\section{BIBLIOGRAPHIE}

Archibugi D., 2009, La démocratie cosmopolitique, Paris, Cerf.

Arrous J., 1994, Introduction à l'économie politique, Paris, Dalloz.

Auby J.-B., 2006, La décentralisation et le droit, Paris, LGDJ, collection Systèmes.

Bachelard G., 1996 (1934), Le Nouvel esprit scientifique, Paris, PUF.

Barbosa J., 2008, L'Amérique latine dans la spirale du néolibéralisme : logique d'empire(s) en action, Paris, L'Harmattan.

Benichi R., 2008, Histoire de la mondialisation, Paris, Vuibert.

Bernard E., 2010, La spécificité du standard juridique en droit communautaire, Bruxelles, Bruylant, collection Droit de l'union européenne, $\mathrm{n}^{\circ} 16$.

Bertrand M.-J., 1974, Géographe de l'Administration. L'impact du pouvoir exécutif dans les capitales nationales, Paris, Éditions M. Th. Génin.

Bodichon Dr., 1866, De l'Humanité, Bruxelles, Lacroix, Verboechhoven \& Cie.

Braudel F., 1979 (2008), La dynamique du capitalisme, Paris, Flammarion, collection Champs Histoire.

Braudel F., 1985 (1993), Civilisation matérielle et capitalisme (XV'e-XVIII ${ }^{e}$ siècles), Paris, Livre de Poche, 3 tomes

Bridel L., 1908, Géographie juridique de l'occident, Japon, Sansaisha.

Brimo A., 1986, « La notion d'espace et le droit », in Mélanges P. Montané de la Roque, Toulouse, PUSS, p .68.

Caillaux J., 1942, Mes Mémoires, Paris, Plon. 
Cappeler W. et Kitamura T. (dir.), 1998, Une introduction aux cultures juridiques non occidentales (autour de Masaji Chiba), Bruxelles, Bruylant, collection bibliothèque de l'Académie européenne de théorie du Droit, $\mathrm{n}^{\circ} 1$.

Cavaillé F., 2009, « Quelles interdisciplinarités entre la géographie et le droit ? Vers une géographie juridique? », in P. Forest (dir.), Géographie du droit. Épistémologie, développement et perspectives, Laval, PUL, collection Diké, p. 45-67.

Cenk Keskin A., 2010, Pour un nouveau droit international de la concurrence, Paris, L'Harmattan, 2010. Chakrabarty D., 2010, La pensée postcoloniale et la différence historique, Paris, Éditions Amsterdam.

Chanda N., 2010, Au commencement était la mondialisation. La grande saga des aventuriers, missionnaires, soldats et marchands, Paris, CNRS Éditions.

Chantebout B., 2011, Droit constitutionnel, Paris, Sirey, collection université, $28^{\mathrm{e}}$ édition. Charvet J.-P. et Sivignon M. (dir.), 2002, Géographie humaine. Questions et enjeux du monde contemporain, Paris, Armand Colin.

Chauveau J.-P., Jul-Larsen E., Chaboud C., 2000, Les pêches piroguières en Afrique de l'Ouest. Dynamiques institutionnelles : pouvoirs, mobilités, marchés, Paris, Éditions Karthala.

Chua A., 2007, Le monde en feu. Violences sociales et mondialisation, Paris, Éditions du Seuil. Cogneau D., 2007, L'Afrique des inégalités : où conduit l'histoire, Paris, Éditions Rue d'Ulm. Coudenhove-Kalergi R. de, 1988, Pan Europe, Paris, PUF, collection publication de l'Institut universitaire d'études européennes.

Dan Zlatescu V., 1981, Geografie juridica contemporana : Introducere in teoria marilor sisteme de drept, Bucarest, Éditions știinȚifica și Enciclopedica

David R. et Jauffret-Spinosi C., 2002, Les grands systèmes de droit contemporains, Paris, collection Précis Droit privé, $11^{\mathrm{e}}$ édition.

David R., 1966, « Géographie juridique », extrait de l’Encyclopédie de la Pléiade, géographie générale, Paris, Gallimard, p. 1738-1748.

Debie F., 1995, Géographie économique et humaine ", Paris, PUF, 1995.

Delmas-Marty M., 2006, Le pluralisme ordonné, Paris, Éditions du Seuil, collection les forces imaginaires du droit, tome 2.

Dorion H., 1980, « La constitution canadienne et les partages géographiques », Cahiers de géographie du Québec, volume 24, nº 61, p. 69-79

Dubus N., Helle C. et Masson-Vincent M., 2010, De la gouvernance à la géogouvernance : De nouveaux outils pour une démocratie locale renouvelée ", L'Espace Politique [En ligne], 10|2010-1, mis en ligne le 15 avril 2010, Consulté le 30 septembre 2011. URL : http://espacepolitique.revues.org/ index1574.html.

Dupont-Ferrier G., 1930, Essai sur la géographie administrative des élections financières en France de 1356 à 1790, Paris, Extrait de l'Annuaire-Bulletin de la Société de l'histoire de France, années 1928 et 1929.

Dupuy C. et Lavigne S., 2009, Géographies de la finance mondialisée, Paris, La documentation française, collection Études, $\mathrm{n}^{\circ} 5299$.

Eisenmann C., 1967 (1982), Cours de droit administratif, Paris, LGDJ. 
Espinas. G., 1931, « Histoire urbaine : directions de recherches et résultats », Annales d'histoire économique et sociale, volume $3, \mathrm{n}^{\circ} 11, \mathrm{p} .394-427$.

Ferreira Da Cunha P.J.F., 2009, Geografia Constitucional, Lisbonne, Quid juris (en portugais).

Ferreira da Cunha P.J.F., 2010, Traité de droit constitutionnel, Constitution universelle et mondialisation des valeurs fondamentales, Paris, Buenos Books America.

Fichter J-H., 1965, La sociologie. Notions de base, Paris, Éditions universitaires, collection encyclopédie universitaire.

Fiero-Domenech A., 1986, Le pré carré. Géographie historique de la France, Paris, Robert Laffont, collection pluriel.

Forest P., 2009, «Introduction », in P. Forest (dir.), Géographie du droit. Épistémologie, développement et perspectives, Laval, PUL, collection Diké, p. 7-20.

Foucault M., 2004, Naissance de la biopolitique, Paris, Gallimard/Éditions du Seuil.

Foucher M., 2007, L'obsession des frontières, Paris, Perrin.

Glenn P., 2010, Legal Traditions in the World. Sustainable Diversity of Law, Oxford, Oxford University Press.

Gottmann J., 1952, La politique des États et leur géographie, Paris, Armand Colin.

Goyheneche E., 1979 (1961), Notre terre basque : notions de géographie, histoire et culture populaire ", Pau, Société nouvelle d'éditions régionales et de diffusion, $2^{\mathrm{e}}$ édition.

Guillebaud J.-C., 2003, Le goût de l'avenir, Paris, Éditions du Seuil, collection Points.

Gyselen R. (dir.), 1989, La géographie administrative de l'Empire Sassanide. Les témoignages sigillographique, Bure-Sur-Yvette, Groupe pour l'étude de la civilisation du Moyen-Orient.

Husa J., 2004, "Classification of Legal Families Today, is it a time for memorial hymn", RIDC, volume $56, \mathrm{n}^{\circ} 1$, p. $11-38$.

Kalampalikis N., 2007, Les grecs et le mythe d'Alexandre, Paris, L'Harmattan, collection logiques sociales.

Kant E., 1988 (1784), Idée d'une histoire universelle au point de vue cosmopolitique, Paris, Bordas.

Legeais R., 2008, Grands systèmes de droit contemporains. Approche comparative, Paris, Litec, collection manuel, $2^{\mathrm{e}}$ édition.

Marseille J., 1984, Empire colonial et capitalisme français. Histoire d'un divorce, Paris, Albin Michel.

Martens P., 2009, « Préface à la première édition », in Corten O. et Schaus A., Le droit comme idéologie. Introduction critique au droit belge, Bruxelles, Éditions de l'Université de Bruxelles, $2^{\mathrm{e}}$ édition revue, p. 7-9.

Mc Luhan M. et Fiore Q., 1967, The Medium is The Massage: An Inventory of Effects, UK, Penguin Books.

Mehl L. et Beltram P., 1984, Science et technique fiscales, Paris, PUF, collection thémis Droit. Montesquieu C-L.S., 1860, Esprit des Lois, Paris, Firmin Didot Frères.

(multi) (1995), Question de : le XXI ${ }^{e}$ siècle a commencé, $\mathrm{n}^{\circ}$ 100, Paris, Albin Michel.

Nerriere J.-P., 2004, Don't speak English... Parler globish!, Paris, Eyrolles.

Nouschi M., 2007, Le XX' siècle. Temps, tournants, tendances, Paris, Albin Colin, coll. U. 
Orsenna E., 2006, Voyage aux pays du coton, Petit précis de mondialisation, Paris, Fayard.

Ould el Hacen M., 1989, « Centre et régions en Mauritanie », Revue du Monde musulman et de la Méditerranée, volume 54, p. 149-160.

Parra P., 2007, « Une approche géographique du terrain juridique : la géographie juridique ou la géographie du Droit », in Cahiers de l'ADES, Approches des terrains de recherches, p. 27-37.

Perret J.-M., 1994, « Pour une géographie juridique », Annales de Géographie, tome 103, n 579, p. 520-526.

Perret J.-P., 2009, « Droit spatialisé et espace juridiquement modelé », in P. Forest (dir.), Géographie du droit. Épistémologie, développement et perspectives, Laval, PUL, collection Diké, p. 261-268.

Pomeranz K., 2010, Une grande divergence. La Chine, L'Europe et la construction de l'économie mondiale, Paris, Albin Michel.

Popper K., 1979, La société ouverte et ses ennemis, Paris, Éditions du Seuil (2 volumes).

Raffestin C., 1985, « Religions, relations de pouvoir et géographie politique », Cahiers de Géographie du Québec, volume. 29, nº 76, 1985, p. 101-107.

Reich R., 1997, L'économie mondialisée, Paris, Dunod.

Revel J.-F., 2002, L'obsession anti-américaine, Paris, Plon.

Ricœur P., 1967, « Violence et langage », in Recherches et Débats (La violence), 16 (1967), n 59, p. 86-94.

Sacco R., 1991, La comparaison juridique au service de la connaissance du droit, Paris, Economica, Études juridiques comparatives.

Saidon A. K.., 1993, Droit comparé et géographie du monde », Moscou, P.A.H. (en russe) (compterendu d'A. Vorobion dans RIDC, 1994, p. 1213).

Sassen S., 2009, Critique de l'État. Territoire, Autorité et Droits, de l'époque médiévale à nos jours, Paris, Le Monde diplomatique/Demopolis.

Schroter F., 2007, Les Frontières de la Suisse : questions choisies, Zurich, Schulthess/Paris, LGDJ/ Bruxelles, Bruylant.

Sy Mouhandou M., 2007, La protection constitutionnelle des droits fondamentaux en Afrique. L'exemple du Sénégal, Paris, L’harmattan, collection Études africaines.

Tarde G., 1993 (1895), Les lois de l'imitation », Paris, Kimé, collection Vues critiques (reproduction en fac-similé de la $2^{\mathrm{e}}$ édition, Paris, Alcan, 1895).

Thiebault J.-L., 2006, « La controverse entre comparatistes et spécialistes des aires culturelles. À propos de la transition vers la démocratie dans les pays postcommunistes », in Mélanges offerts au Professeur M. Lesage, Paris, Société de Législation Comparée, p. 327-343.

Thual F., 2009, « Pour une génétique des frontières ", Géopolitiques, n 104, janvier, p. 46-48.

Todd E., 2011, L'origine des systèmes familiaux, Paris, Gallimard, tome 1 : « L'Eurasie ».

Troper M., « Système de droit et État », in F. Terre (dir.), Le système juridique, Archives du droit, Paris, Sirey, 1986, tome 31, p .28.

Virilio P., 2007, L'université du désastre, Paris, Galilée, collection L'espace critique. 
Wald A., 2005, « Doit-on repenser les "familles juridiques" ? », in Mélanges X. Blanc-Jouvan, Paris, Société de Législation comparée, p. 187-200.

Wallon Leducq C-M. et al., 1988, Frontières économiques, administratives et politiques, Lille, Les cahiers du CRAPS, $n^{\circ} 4$, février.

Wijffels A., 2008, « Le droit comparé à la recherche d'un nouvel interface entre ordres juridiques ", Revue de droit international et de droit comparé, volume 85, n²-3, p. 228-252.

\section{RÉSUMÉS}

La rencontre entre géographie et droit est ancienne et chacun est tenté de classifier ses données géographiques en groupes et sous-groupes. Cette entreprise s'avère être pourtant difficile dans une économie politique mondialisée, de telle sorte que la génétique juridique, programme certes plus ambitieux, permettrait de comprendre et de définir la géographique juridique comme une simple idéologie.

The confluence of geography and law is very old and everyone has tried to classify its geography data in groups and sub-groups. That initiative look like difficulty in a globalized political economics, in such a way the inherited legal would help to understand and to define the "legal geography" as a simple ideology.

\section{INDEX}

Keywords : legal geography, legal inherited, legal, political economics, globalization

Mots-clés : géographie, droit, génétique juridique, économie politique, mondialisation

\section{AUTEUR}

\section{JEAN-FRANÇOIS BOUDET}

Jean-François Boudet, maître de conférences (HDR) en Droit public à la Sorbonne Paris Cité, Université Paris Descartes ; membre du Centre d'Études et de Recherches de Sciences Administratives et Politiques (CERSA - CNRS - Université Paris II UMR 7106) et collaborateur scientifique au Centre Montesquieu d'Action publique (Université catholique de Louvain). Il est membre élu du conseil d'administration de la Société française de Finances publiques (SFFP), collaborateur scientifique au CMAP (Université catholique de Louvain) et au Tax Institute (Université libre de Liège). Ses champs de recherches concernent les Institutions financières publiques, jean-francois.boudet@paris.descartes.fr 